\title{
Effects of alpha-glucosidase-inhibiting drugs on acute postprandial glucose and insulin responses: a systematic review and meta-analysis
}

\author{
Marjan Alssema ${ }^{1,2}$, Carolien Ruijgrok², Ellen E. Blaak $\mathbb{B}^{3}$, Léonie Egli ${ }^{4}$, Pierre Dussort ${ }^{5}$, Sophie Vinoy ${ }^{6}$, \\ Jacqueline M. Dekker ${ }^{2}$ and M. Denise Robertson ${ }^{7}$
}

\begin{abstract}
Background/objectives: Despite considerable literature supporting the potential health benefits of reducing postprandial glucose (PPG), and insulin (PPI) exposures, the size of a clinically relevant reduction is currently unknown. We performed a systematic review and meta-analysis to quantify effects of alpha-glucosidase-inhibiting (AGI) drugs on acute PPG and PPI responses.

Methods: We searched EMBASE and MEDLINE until March 13, 2018 for controlled studies using AGl drugs together with a standardized carbohydrate load or mixed meal. The mean incremental PPG and PPI levels were calculated as outcomes. Meta-analyses, stratified by diabetes state, were performed by using random effects models.

Results: The 66 included publications comprised 127 drug-control comparisons for PPG, and 106 for PPI, mostly testing acarbose or miglitol. The absolute effects on PPG were larger among individuals with diabetes $(-1.5 \mathrm{mmol} / \mathrm{l}$ mean PPG $[95 \% \mathrm{Cl}-1.9,-1.1]$ by acarbose, and $-1.6[-1.9,-1.4]$ by miglitol) as compared to individuals without diabetes $(-0.4[95 \% \mathrm{Cl}-0.5,-0.3]$ by acarbose, and $-0.6[-0.8,-0.4]$ by miglitol). Relative reductions in PPG by both drugs were similar for diabetic and non-diabetic individuals (43-54\%). Acarbose and miglitol also significantly reduced mean PPI, with absolute and relative reductions being largest among individuals without diabetes.
\end{abstract}

Conclusions: The present meta-analyses provide quantitative estimates of reductions of PPG and PPI responses by AGl drugs in diabetes and non-diabetic individuals. These data can serve as benchmarks for clinically relevant reductions in PPG and PPI via drug or diet and lifestyle interventions.

\section{Introduction}

Elevated glucose levels in the postprandial state are a key feature of impaired glucose tolerance and diabetes ${ }^{1}$ and are a risk marker for cardiovascular diseases $(\mathrm{CVD})^{2}$. Reducing exposure to high glucose levels in the postprandial state is therefore a target for diabetes patients, as

Correspondence: Pierre Dussort (publications@ilsieurope.be)

${ }^{1}$ Former employee of Unilever Research and Development, Vlaardingen, The Netherlands

${ }^{2}$ Department of Epidemiology and Biostatistics, VU University Medical Center, Amsterdam Public Health Research Institute, Amsterdam, The Netherlands

Full list of author information is available at the end of the article emphasized in guidelines from the International Diabetes Federation, and the American Diabetes Association ${ }^{3,4}$. Increments in postprandial glucose (PPG) levels are generally considered as risk factors for micro and macrovascular complications via several pathways ${ }^{5,6}$. Therefore, PPG lowering is an important route for reducing chronic disease risk, also for the general population (at risk) ${ }^{5}$.

Direct evidence for the beneficial effects of reducing PPG comes from studies with alpha-glucosidase-inhibiting (AGI) drugs (acarbose, miglitol, voglibose). These drugs, which primarily act to lower PPG by slowing down the rate of carbohydrate digestion and gastrointestinal glucose uptake,

\section{(c) The Author(s) 2021}

(c) (i) Open Access This article is licensed under a Creative Commons Attribution 4.0 International License, which permits use, sharing, adaptation, distribution and reproduction cc) in any medium or format, as long as you give appropriate credit to the original author(s) and the source, provide a link to the Creative Commons license, and indicate if changes were made. The images or other third party material in this article are included in the article's Creative Commons license, unless indicated otherwise in a credit line to the material. If material is not included in the article's Creative Commons license and your intended use is not permitted by statutory regulation or exceeds the permitted use, you will need to obtain permission directly from the copyright holder. To view a copy of this license, visit http://creativecommons.org/licenses/by/4.0/. 
have been shown to improve glycaemic control ${ }^{7}$ and reduce type 2 diabetes mellitus (T2DM) risk ${ }^{8,9}$. Long-term effects on CVD outcomes have been inconsistent ${ }^{9,10}$. In support of the benefits of reducing PPG, consumption of diets with a lower glycemic index (GI) or load have been associated with lower risk of coronary heart disease $(\mathrm{CHD})^{11}$, and $\mathrm{T}_{2} \mathrm{DM}^{12,13}$. Furthermore, interventions with lower GI diets may improve glycaemic control and insulin sensitivity in particular in diabetes ${ }^{14,15}$.

Despite considerable literature supporting the likely health benefits of reducing PPG exposures, the size of PPG reduction that is needed to translate into a meaningful reduction in disease risk (factors) is currently unknown. Dietary trials with lower GI diets generally lack precise quantification of glycaemic exposure in real life ${ }^{5}$. AGI drugs have been approved for the treatment of diabetes since the $1990 \mathrm{~s}^{16}$. These drugs have been studied for both acute postprandial effects and longer-term effects on risk factors. Meta-analyses of studies on effects of these AGIs have estimated these produce clinically relevant reductions of glycated haemoglobin (HbA1c) levels between -0.5 and $-1.5 \%(5-16 \mathrm{mmol} / \mathrm{mol})$ in subjects with diabetes ${ }^{17-20}$. Given the proven clinical efficacy of AGI drugs, their quantitative effects on PPG and postprandial insulin (PPI) could serve as a benchmark for interpreting the potential health relevance of the changes in PPG and PPI by other drugs or lifestyle interventions. However, to date, the acute postprandial effects of alphaglucosidase inhibitors on glucose and insulin responses after a carbohydrate-containing meal have never been systematically reviewed and quantified.

Therefore, the aim of this review was to systematically review the quantitative effects of alpha-glucosidase inhibitors on acute PPG and PPI responses.

\section{Materials and methods}

\section{Protocol, data source and search}

PRISMA reporting guidelines were followed, and the protocol was registered in the International prospective register of systematic reviews (PROSPERO, number CRD42018085522). Electronic databases (Elsevier Medical Database (EMBASE) and the US National Library of Medicine database (MEDLINE via the PubMed portal) were used to search for relevant papers until March 13, 2018. The full search string can be found in Supplementary Data 1 . The search was designed to identify controlled trials with AGI drugs together with a standardized carbohydrate load or mixed meal, studying effects on acute PPG and/or PPI responses.

\section{Selection of relevant studies}

All titles and abstracts were screened in duplicate by two authors (M.A. and J.M.D.) and differences were resolved by consensus. Full-text publications were subjected to detailed examination against inclusion criteria by couples of two researchers (M.A., C.R., J.M.D., L.E., M.D.R.). Differences in the provisional inclusion or exclusion of studies by the two researchers were resolved by consultation. The following criteria were applied for inclusion: all populations, including healthy, pre-diabetes and diabetes (both type 1 and type 2 diabetes mellitus), use of AGI drug together with a standardized carbohydrate containing load, control treatment with placebo or no drug, and PPG or PPI as outcome. Exclusion criteria were not English language, oral glucose as carbohydrate load, no control treatment, co-intervention (an additional intervention in treatment arm but not the control arm), comparative study (with other drugs), and individuals who have undergone gastric surgery. Studies with insulin administration during the test meals were included for analyses of PPG outcomes, but excluded for analyses of insulin outcomes. Multiple arms of the same study were included when multiple arms were independent (had different control groups) ${ }^{21}$. Treatments arms with the same control group were included only if the arms were in different (subgroup) analyses.

\section{Data extraction and quality assessment}

Data from included publications were extracted by one researcher (M.A.) and a random subsample of $10 \%$ was checked by another researcher (C.R.). Information on the study design, population, drug, dosage, test meal and outcome measures (glucose and insulin incremental, or total area under the curve (AUC), mean postprandial level, variance or $p$-values) were extracted. If no AUC or mean postprandial level was reported, postprandial response data per timepoint were extracted. Data from figures (i.e. bars for AUC, and responses per timepoint from graphs) were extracted using the Microsoft Excel add-in tool TM Image-to-data (tushar-mehta.com). If studies reported responses to multiple sequential meals, data from responses to the first meal were extracted. If the study duration was multiple days, data from day 1 were used.

The study quality was assessed with the Cochrane Collaboration's tool for assessing risk of bias by scoring seven different items (random sequence generation, allocation concealment, blinding of participants and personnel, blinding of outcome data, incomplete outcome data, selective reporting, other bias) ${ }^{21}$. The item 'blinding of outcome data' was universally scored as 'low risk' because glucose and insulin are outcome measures than can be objectively assessed.

\section{Data synthesis and analysis}

Glucose and insulin incremental or total AUCs, with variance measure, were transformed into SI units $(\mathrm{mmol} / \mathrm{l}$ for glucose $(=0.0555 \mathrm{mg} / \mathrm{dl})$ and $\mathrm{pmol} / \mathrm{l}$ for insulin $(=6 \mathrm{microU} / \mathrm{ml})$ ). AUCs were divided by time (the duration of the postprandial measurement period) to express outcomes as average postprandial levels (for tAUC) or average 
postprandial increase (for iAUC). Standard errors (SE) were transformed into standard deviations $(\mathrm{SD})(\mathrm{SE}=\mathrm{SD} / \sqrt{ } N$, where $N=$ subject population). Where only $p$-values were reported, these were used to estimate the $\mathrm{SE}^{21}$. In cases where responses were only reported as data per timepoint (in Table or as a Figure), incremental AUC's were calculated by the trapezoidal method as net incremental $\mathrm{AUC}^{22}$. The calculation of the variance of the iAUC was based on SDs of individual timepoints by using matrix algebra involving a covariance matrix with the assumed correlation structure being compound symmetry ${ }^{23}$. Assumed betweentimepoints correlation were $r=0.75$ for glucose and $r=0.5$ for insulin. Meta-analysis was performed in Review Manager (RevMan) ([Computer program], version 5.3. Copenhagen: The Nordic Cochrane Centre, The Cochrane Collaboration, 2014). Random effects models with inverse variance weighing were used to estimate the combined absolute effects, which is expressed as a difference in average postprandial increase. Relative changes were calculated as $100 \% \times\left(\mathrm{iAUC}_{\text {drug }}-\mathrm{iAUC}_{\text {control }}\right) /\left(\mathrm{iAUC}_{\text {control }}\right)$, with $\mathrm{SE}$ of relative change calculated as earlier described ${ }^{24}$, assuming a within subject correlation coefficient of 0.7 . Heterogeneity and subgroup differences were assessed by $I^{2}$ and $\mathrm{Chi}^{2}$ statistic $(p<0.05)$, respectively. Fixed effects models were run as sensitivity analyses.

Main analyses were conducted for each drug and in subgroups of dosage, for diabetes and non-diabetic individuals separately. Other subgroup analyses were performed if there were a minimum of three comparisons per subgroup, and these were: duration of postprandial measurement period, timing of drug administration (before or with meal), mixed meal versus carbohydrate meal ( $\leq 70$ vs. $>70 \% \mathrm{E}$ ), and carbohydrate content of the meal.

\section{Results}

\section{Characteristics of included studies}

The search retrieved 1811 publications and an additional 15 potentially relevant publications were found manually and added to the database for screening (Fig. 1). After removal of duplicates, 1341 records were screened based on titles and abstracts; 176 full-text publications were finally assessed for eligibility.

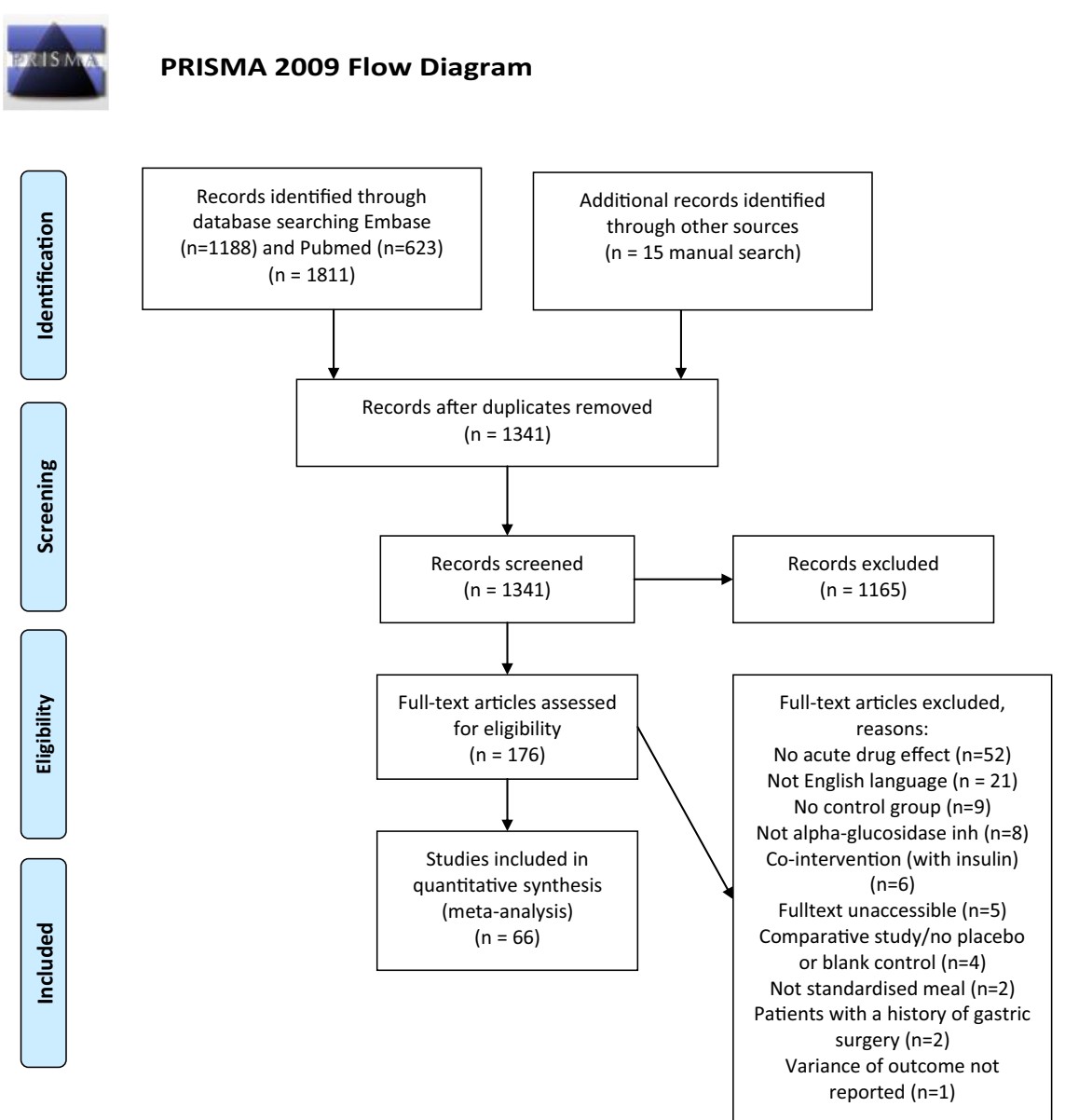

Fig. 1 PRISMA flow diagram of literature search: PRISMA=preferred reporting items for systematic reviews and meta-analyses. PRISMA flow-diagram. 
The 66 included publications (Supplementary Table 1) comprised 134 comparisons, of which 127 comparisons had information on PPG and 106 comparisons had information on PPI. For PPG, the number of comparisons per drug was 64, 48, 6 and 8 for acarbose, miglitol, voglibose and emiglitate, respectively. For PPI, the number of comparisons per drug was 46, 42, 10 and 8 for acarbose, miglitol, voglibose and emiglitate, respectively. Most of the comparisons were among individuals without diabetes (84 out of 134) (Supplementary Table 1). Of the 50 comparisons among patients with diabetes, 6 were among type 1 diabetes and 44 among type 2 diabetes. The range in duration of postprandial measurement was $90-360 \mathrm{~min}$, with a median and most frequent duration of $180 \mathrm{~min}$. Standardized meals were mostly mixed meals (90 out of 127 comparisons), others were high carbohydrate loads (>70\% E carbohydrates). Total energy content of standardized meals ranged from 200 to $900 \mathrm{kcal}$, and carbohydrate content ranged from 32 to $125 \mathrm{~g}$ (Supplementary Table 1).

\section{Acarbose and miglitol-main findings}

Acarbose and miglitol significantly reduced mean PPG among individuals with and without diabetes (Table 1). The absolute effects of acarbose and miglitol on PPG were larger among individuals with diabetes $(-1.5 \mathrm{mmol} / \mathrm{l}$ mean PPG [95\% CI $-1.9,-1.1]$ by acarbose, and $-1.6[-1.9$, -1.4 ] by miglitol) as compared to individuals without diabetes $(-0.4 \mathrm{mmol} / \mathrm{l}$ mean PPG [95\% CI $-0.5,-0.3$ ] by acarbose, and $-0.6[-0.8,-0.4]$ by miglitol $)\left(\mathrm{Chi}^{2}\right.$ for subgroup difference $p<0.05$ for both acarbose and miglitol). Relative effects of both drugs on PPG were similar for diabetic and non-diabetic individuals with a percentage change by acarbose of $-43 \%$ in both groups and by miglitol of $-54 \%$ among individuals without diabetes and $-50 \%$ among individuals with diabetes (Table 1 ).

Acarbose and miglitol also significantly reduced mean PPI among individuals with diabetes $(-38.2 \mathrm{pmol} / \mathrm{l}[95 \%$ CI $-53.3,-23.2]$ by acarbose, and $-12.2[-22.6,-1.7]$ by miglitol) and without diabetes $(-66.7[-97.1,-36.3]$ by acarbose, and $-68.6[-95.2,-42.0]$ by miglitol (Table 1$)$ ). In contrast to effects seen on PPG, absolute and relative reductions on PPI by both drugs were generally larger among individuals without diabetes $\left(\mathrm{Chi}^{2}\right.$ for subgroup difference $p<0.05$ for all subgroup differences except for absolute reduction on PPI by acarbose (Table 1)).

In sensitivity analyses with fixed, instead of random effects models, results were largely similar to random effects model, except for a smaller effect of miglitol on PPI in healthy individuals (Supplementary Table 2).

\section{Acarbose and miglitol-subgroup analyses}

Subgroup analyses with dose revealed that there were no differences between dosages for acarbose (based on studies in non-diabetics only) (Table 2 and Supplementary Fig. 1),

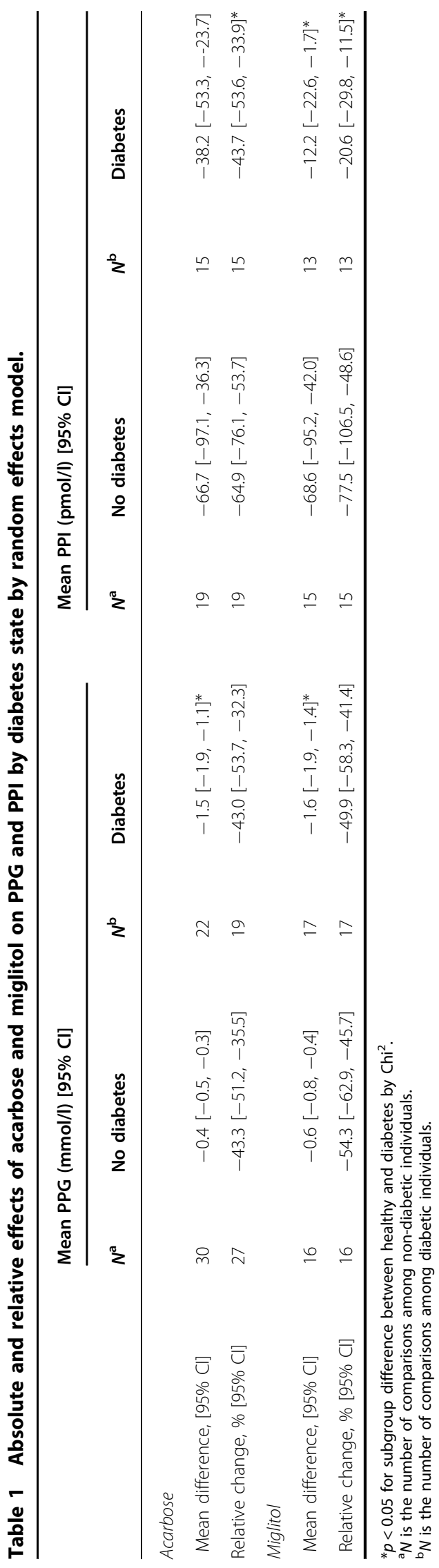




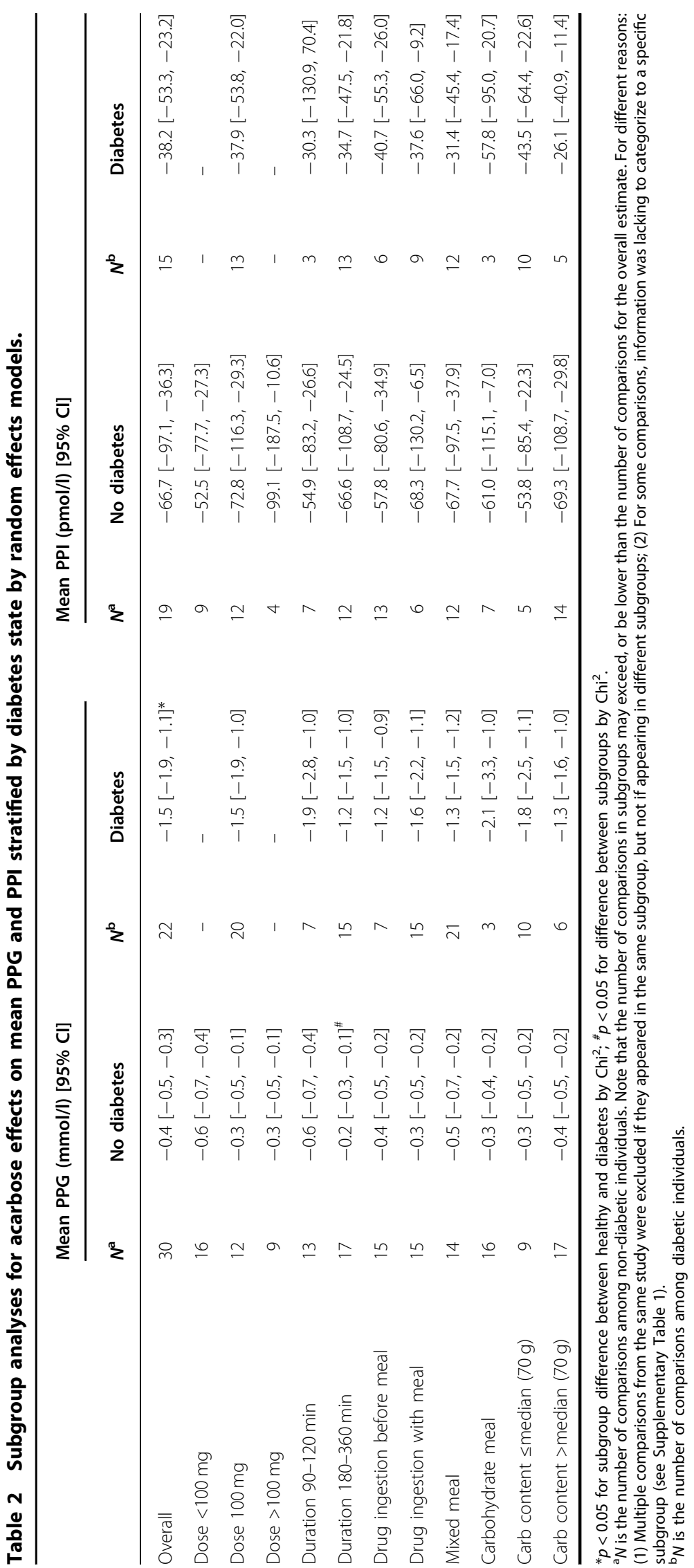


but effects of miglitol on PPG were greater with increasing dose (Table 3 and Supplementary Fig. 1). There were no dose-effects of either drug on PPI. Further subgroup analyses showed that effects of acarbose on PPG were larger with a shorter measurement duration (120 min or less), among individuals without diabetes only (Table 2). Effects of miglitol on PPI were larger with shorter duration and were strongest when the drug was ingested before (as opposed to with) meal, among individuals with diabetes only (Table 3). Effects of miglitol on PPG were larger with lower carbohydrate content $(<70 \mathrm{~g})$, among individuals with diabetes only (Table 3). Finally, effects of miglitol on PPI were larger when consumed with high carbohydrate content (individuals without diabetes only) (Table 3).

\section{Voglibose and emiglitate}

The overall effect of voglibose on PPG and PPI (based on four and five comparisons in predominantly nondiabetic individuals) was not significant (mean difference [95\% CI $]-0.3[-0.6,0.01]$ and $-30.8[-76.9,15.3]$ for PPG and PPI, respectively). The overall effect of emiglitate (based on four comparisons each) was a reduction in PPG and PPI (mean difference [95\% CI $]-0.3[-0.5,-0.03]$ and $-44.3[-66.1,-22.5]$ for PPG and PPI, respectively) (Forest plots with study quality assessments are given in Supplementary Fig. 1).

\section{Study quality assessment}

The risk of bias was low for items blinding of outcome data, incomplete outcome data, selective reporting and other biases most studies. However, the information about the quality items randomization, allocation concealment, and blinding of participants and personnel was lacking or incomplete in most studies (Supplementary Fig. 1).

\section{Discussion}

This is the first systematic investigation aiming to quantify the effects of AGI drugs on acute PPG and PPI responses. As expected, AGI drugs reduce PPG and PPI responses among individuals with and without diabetes, with reductions in incremental PPG of $\sim 45-50 \%$ and of 20-75\% in incremental PPI. Absolute effects on PPG were largest among diabetes patients, but relative effects were comparable for individuals with and without diabetes. Absolute and relative effects on PPI were larger among individuals without diabetes. Effects of acarbose were not dose-dependent, but effects of miglitol on PPG were larger with increasing dose.

The effects of AGI drugs on PPG and PPI are well known from their primary mechanism of action, which is the slowing down of carbohydrate digestion ${ }^{16}$. The present meta-analysis shows a larger absolute effect of acarbose and miglitol on PPG among individuals with versus without diabetes, most likely because the higher overall
PPG response in the former. Indeed, while absolute reductions in PPG were larger in that group, relative reductions were comparable between individuals with and without diabetes. Previous studies do not provide strong indications that differences in rates of absorption contribute to the greater absolute effects of AGI's in diabetes. Indeed, faster, but also slower or equal, rates of gastric emptying have been observed in obesity and diabetes, and data on this are not conclusive ${ }^{25}$. Data from a stable isotope study show comparable rates of glucose absorption in individuals with and without diabetes ${ }^{26}$.

In contrast to the stronger absolute effects on PPG among individuals with diabetes, the reduction in PPI was largest among non-diabetic individuals. Patterns of insulin secretion in diabetes patients have been shown to be more irregular and not closely linked to glucose responses ${ }^{27}$. The reduction of PPI in healthy volunteers is probably the result of the rapid adaptation of insulin secretion to reduced PPG responses, which is not optimal in diabetes patients.

An overall dose-response relationship with PPG lowering was observed for miglitol but not acarbose (based on studies in non-diabetics only). The lack of a dose-response effect for acarbose is in line with the within-study effects of underlying studies; most of the included studies do not provide indications for dose-response effects ${ }^{28-35}$. In meta-analyses of longerterm studies with acarbose in diabetes, there was no dosedependent effect seen on $\mathrm{HbA1} \mathrm{c}^{17,19}$, but there was for post-load glucose ${ }^{17}$. The observed dose-response effect for miglitol on PPG is in line with the within-study comparisons in underlying studies, most though not all of which indicate dose-dependent effects of miglitol on $\mathrm{PPG}^{36-39}$. Moreover, longer-term studies, although scarce, have been suggestive of a dose-response effect of miglitol on $\mathrm{HbA} 1 \mathrm{c}^{17}$.

Effects of AGI drugs on responses to mixed meals vs. carbohydrate meals, and high vs. low carbohydrate loads, did not provide support for the hypothesis that AGIs are most effective when accompanying high carbohydrate meals. Asian diets are generally higher in carbohydrate content, but a meta-analysis on effects of AGIs in Asian versus non-Asian populations did not reveal differential effects on glycaemic control ${ }^{20}$. However, we cannot fully rule out differential effects dependent on meal composition because the studies with high carbohydrate content were most often mixed meals, and the number of studies was too small to differentiate for both meal composition and carbohydrate content.

Our analysis indicates that AGI drugs under standardized acute conditions reduce incremental PPG and PPI by $\sim 45-50 \%$ and $\sim 20-75 \%$, respectively. Based on previous studies on the longer-term effects of use of AGIs, achievement of such acute changes under standardized 


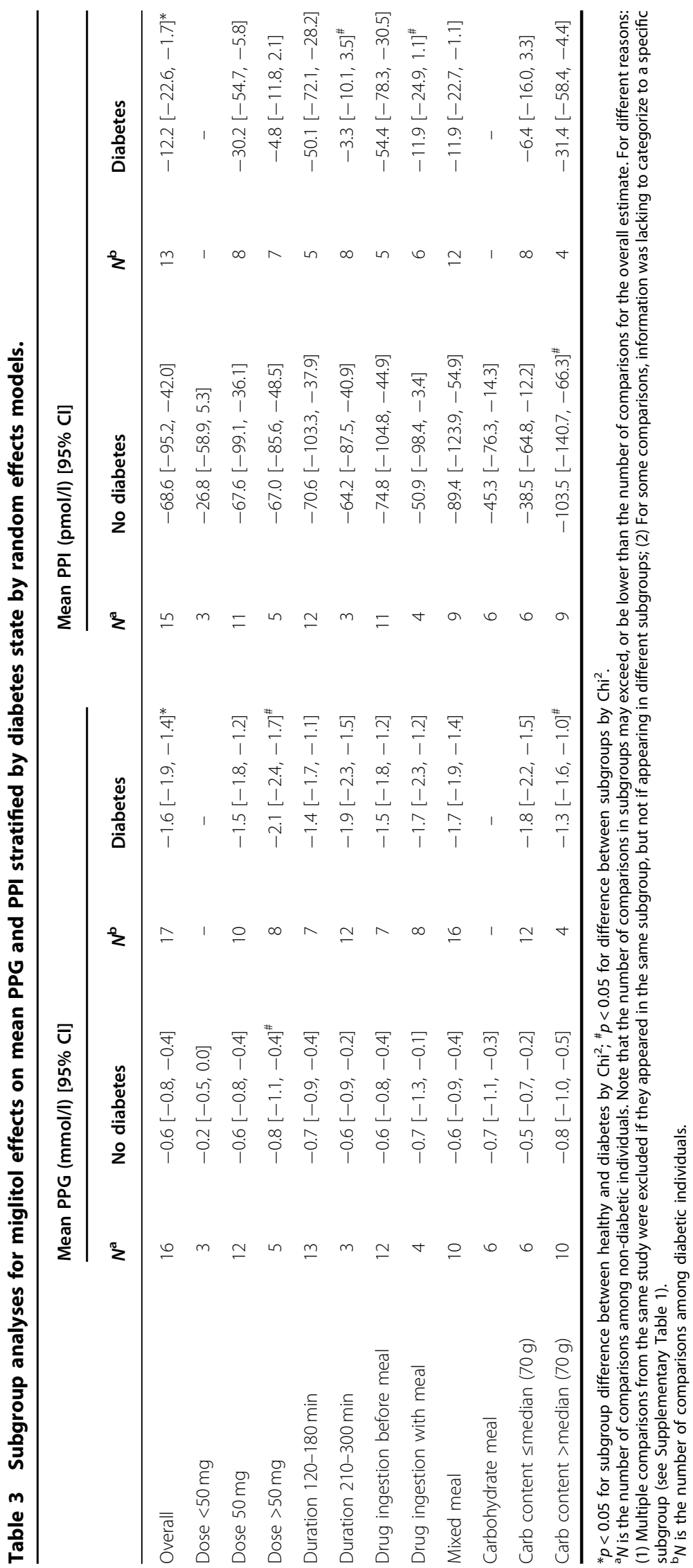


conditions can be expected to lead to clinically relevant chronic effects over time. Previous meta-analyses of studies with AGIs have estimated clinically relevant reductions of HbA1c between -0.5 and $-1.5 \%$ in subjects with diabetes ${ }^{17-20}$. Moreover, the limited number of long-term studies (of 1 year or longer) with AGIs in individuals at increased risk for diabetes is indicative of a reduction in risk of development of overt diabetes ${ }^{9,40}$. However, clinically relevant effects on PPG and PPI should always be considered in the context of the mechanisms of action of the intervention and the metabolic effects beyond PPG and PPI. AGIs as a class reduces carbohydrate digestion, glycaemic variability, lipids, blood pressure, coagulation factors, and have impact on incretin hormones and gut microbiota ${ }^{16}$. Other diet, lifestyle or drug intervention that lower PPG, will also impact a range of upstream and downstream effects, which should be considered when estimating longer-term effects on risk factors or disease outcomes.

The reduction of incremental PPG of $\sim 45-50 \%$, or $0.5 \mathrm{mmol} / \mathrm{l}$ mean PPG (non-diabetes) and $1.5 \mathrm{mmol} / \mathrm{l}$ (in diabetes) as achieved by AGIs may be regarded as a point of reference, rather than a threshold per se. Lower reductions in PPG might lead to relevant changes in metabolic risk factors over time, depending on the related metabolic effects. Indeed, low glycaemic index diets exert improvements in glycaemic control and insulin sensitivity $^{14,15}$, while the reductions in PPG and PPI achieved by such diets may be smaller than the effects of drugs. However, it should be noted that a direct quantification of diets effects on PPG and PPI is largely lacking ${ }^{5}$.

A strength of this study is the comprehensive set of studies included because data from all studies identified as eligible according to inclusion and exclusion criteria were extracted and included in the analyses. A limitation is the lack of standardization in PPG and PPI responses, as different studies have applied different measurement durations and higher or lower frequency of measurements during the postprandial response. This may have led to a greater uncertainty around effect sizes and hence reduced the statistical power to identify effects and differences in effects between subgroups. In addition, the accurate assessment of the PPG/PPI response is ultimately related to the number of time points included in the individual studies. Two of the papers ${ }^{29,41}$ included in our analysis were from studies with only three time points; however, the response to these interventions was within the range reported of other studies.

In summary, the present meta-analyses provide quantitative estimates of effects of AGI drugs on PPG and PPI responses. Absolute reductions in PPG are larger among individuals with diabetes, but reductions in PPI are larger among non-diabetic individuals. These data can serve as benchmarks for clinically relevant reductions in PPG and PPI via drug or diet and lifestyle interventions.

\section{Acknowledgements}

We thank Linda Schoonmade from VU University Amsterdam for designing and executing the literature search strategy. We thank Dr. Matthieu Flourakis for his support on finalising the project.

\section{Author details}

${ }^{1}$ Former employee of Unilever Research and Development, Vlaardingen, The Netherlands. ${ }^{2}$ Department of Epidemiology and Biostatistics, VU University Medical Center, Amsterdam Public Health Research Institute, Amsterdam, The Netherlands. ${ }^{3}$ Department of Human Biology, NUTRIM, School of Nutrition and Translational Research in Metabolism, Maastricht University, Maastricht, The Netherlands. ${ }^{4}$ Former employee of Nestle Research Center, Lausanne, Switzerland. ${ }^{5}$ ILSI Europe, Brussels, Belgium. ${ }^{6}$ Mondēlez International R\&D, Nutrition Department, Saclay, France. ${ }^{7}$ Department of Nutritional Sciences, University of Surrey, Guildford, UK

\section{Author contributions}

M.A., P.D., M.D.R., E.B., L.E., S.V. and J.M.D. designed the study; M.A., J.M.D. and P. D. designed the search strategy; M.A., J.M.D. and C.R. screened all titles and abstracts; M.A., C.R., J.M.D., L.E., and M.D.R. examined full text publications against inclusion criteria; M.A. extracted the data from the included publications; C.R. randomly checked $10 \%$ of the extracted data; M.A. analyzed the extracted data; M.A. wrote the manuscript; all authors edited and commented on a version of the manuscript.

\section{Conflict of interest}

M.A. was an employee of Unilever, a manufacturer of consumer food products; L.E. was an employee of Nestec SA; and SV is an employee of Mondēlez International R\&D; E.E.B., J.M.D., P.D., C.R. and M.D.R. have nothing to disclose. This work was conducted by an expert group of the European branch of the International Life Sciences Institute, ILSI Europe. The Dietary Carbohydrates Task Force coordinated this publication. Industry members of this task force are listed on ILSI Europe's website at http://ilsi.eu/task-forces/nutrition/dietarycarbohydrates/. Experts are not paid for the time spent on this work; however, the non-industry members within the expert group were offered support for travel and accommodation costs from the Dietary Carbohydrates Task Force to attend meetings to discuss the manuscript and a small compensatory honorarium with the option to decline. The expert group carried-out the work that is collecting/analyzing data/information and writing the scientific paper separate to other activities of the task force. The research reported is the result of a scientific evaluation in line with ILSI Europe's framework to provide a precompetitive setting for public-private partnership. ILSI Europe facilitated scientific meetings and coordinated the overall project management and administrative tasks related to the completion of this work. The opinions expressed herein and the conclusions of this publication are those of the authors and do not necessarily represent the views of ILSI Europe nor those of its member companies or any regulatory authority.

\section{Publisher's note}

Springer Nature remains neutral with regard to jurisdictional claims in published maps and institutional affiliations.

Supplementary information The online version contains supplementary material available at https://doi.org/10.1038/s41387-021-00152-5.

Received: 8 May 2019 Revised: 11 June 2020 Accepted: 3 February 2021 Published online: 03 March 2021

\footnotetext{
References

1. World Health Organisation. Definition and diagnosis of diabetes mellitus and intermediate hyperglycemia: report of a WHO/IDF consultation. (WHO, 2006).

2. Levitan, E. B., Song, Y., Ford, E. S. \& Liu, S. Is nondiabetic hyperglycemia a risk factor for cardiovascular disease? Arch. Intern. Med. 164, 2147-2155 (2004).
} 
3. International Diabetes Federation Guideline Development Group, Guideline for management of postmeal glucose in diabetes. Diabetes Res Clin Pract. 103, 256-268 (2014).

4. American Diabetes Association. 6. Glycemic targets: standards of medical care in diabetes. Diabetes Care 41, S55-S64 (2018).

5. Blaak, E. E. et al. Impact of postprandial glycaemia on health and prevention of disease. Obes. Rev. 13, 923-984 (2012).

6. Ceriello, A., Colagiuri, S., Gerich, J. \& Tuomilehto, J. Guideline for management of postmeal glucose. Nutr. Metab. Cardiovasc. Dis. 18, S17-S33 (2008).

7. Van de Laar, F. A. et al. Alpha-glucosidase inhibitors for type 2 diabetes mellitus. Cochrane Database Syst. Rev. 2, CD003639 (2005).

8. Chiasson, J. -L. et al. Acarbose for prevention of type 2 diabetes mellitus: the STOP-NIDDM randomised trial. Lancet 359, 2072-2077 (2002).

9. Holman, R. R. et al. Effects of acarbose on cardiovascular and diabetes outcomes in patients with coronary heart disease and impaired glucose tolerance (ACE): a randomised, double-blind, placebo-controlled trial. Lancet Diabetes Endocrinol. 5, 877-886 (2017).

10. Nauck, M. A. \& Meier, J. J. Break point instead of ACE: acarbose, post-load glycaemic excursions, and cardiovascular events. Lancet Diabetes Endocrinol. 5, 843-845 (2017)

11. Barclay, A. W. et al. Glycemic index, glycemic load, and chronic disease riska meta-analysis of observational studies. Am. J. Clin. Nutr. 87, 627-637 (2008).

12. Livesey, G., Taylor, R., Livesey, H. \& Liu, S. Is there a dose-response relation of dietary glycemic load to risk of type 2 diabetes? Meta-analysis of prospective cohort studies. Am. J. Clin. Nutr. 97, 584-596 (2013).

13. Dong, J. Y., Zhang, L., Zhang, Y. H. \& Qin, L. Q. Dietary glycaemic index and glycaemic load in relation to the risk of type 2 diabetes: a meta-analysis of prospective cohort studies. Br. J. Nutr. 106, 1649-1654 (2011).

14. Brand-Miller, J. C., Hayne, S., Petocz, P. \& Colagiuri, S. Low-glycaemic index diets in the management of diabetes. Diabetes Care 26, 2261-2267 (2003).

15. Livesey, G., Taylor, R., Hulshof, T. \& Howlett, J. Glycemic response and healtha systematic review and meta-analysis: relations between dietary glycemic properties and health outcomes. Am. J. Clin. Nutr. 87, 258S-268S (2008).

16. Joshi, S. R. et al. Therapeutic potential of alpha-glucosidase inhibitors in type 2 diabetes mellitus: an evidence-based review. Expert Opin. Pharmacother. 16, 1959-1981 (2015).

17. Van de Laar, F. A. L. et al. Alpha-glucosidase inhibitors for type 2 diabetes mellitus. Cochrane Database Syst. Rev. CD003639 (2005) https:/doi.org/ 10.1002/14651858.CD003639.pub2.

18. Cai, X., Han, X., Luo, Y. \& Ji, L. Comparisons of the efficacy of alpha glucosidase inhibitors on type 2 diabetes patients between Asian and Caucasian. PLOS ONE 8, e79421 (2013).

19. Zhu, Q., Tong, Y., Wu, T., Li, J. \& Tong, N. Comparison of the hypoglycemic effect of acarbose monotherapy in patients with type 2 diabetes mellitus consuming an Eastern or Western diet: a systematic meta-analysis. Clin. Ther. 35, 880-899 (2013).

20. Gao, X. et al. Meta-analysis and critical review on the efficacy and safety of alpha-glucosidase inhibitors in Asian and non-Asian populations. J. Diabetes Investig. 9, 321-331 (2018).

21. Higgins J. P. T. et al. (eds). Cochrane Handbook for Systematic Reviews of Interventionsversion. Cochrane, (2020) www.training.cochrane.org/handbook.

22. Brouns, F. et al. Glycaemic index methodology. Nutr. Res. Rev. 18, 145-171 (2005).

23. Johnson, R. A. \& Wichern, D. W. Applied Multivariate Statistical Analysis 4th edn Prentice-Hall, New York. (1998) https://doi.org/10.2307/2533879.
24. Ras, R. T. et al. Consumption of plant sterol-enriched foods and effects on plasma plant sterol concentrations-a meta-analysis of randomized controlled studies. Atherosclerosis 230, 336-346 (2013).

25. Muller, M., Canfora, E. E. \& Blaak, E. E. Gastrointestinal transit time, glucose homeostasis and metabolic health: modulation by dietary fibers. Nutrients $\mathbf{1 0}$, 275 (2018).

26. Basu, A. et al. Effects of type 2 diabetes on insulin secretion, insulin action, glucose effectiveness, and postprandial glucose metabolism. Diabetes Care $\mathbf{3 2}$, 866-872 (2009).

27. Polonsky, K. S. et al. Abnormal patterns of insulin secretion in non-insulindependent diabetes mellitus. N. Engl. J. Med. 318, 1231-1239 (1988).

28. Aziz, T. A., Marouf, B. H., Ahmed, Z. A. \& Hussain, S. Effect of Benfotiamine and silibinin on postprandial hyperglycaemia of healthy subjects challenged with sucrose load: comparative study with acarbose. Int. J. Pharm. Pharm. Sci. 5, 530-533 (2013).

29. DeLeon, M. J., Chandurka, V., Albert, S. G. \& Mooradian, A. D. Glucagon-like peptide-1 response to acarbose in elderly type 2 diabetic subjects. Diabetes Res Clin. Pract. 56, 101-106 (2002).

30. Hillebrand, I., Boehme, K., Frank, G., Fink, H. \& Berchtold, P. The effects of the alpha-glucosidase inhibitor BAY g 5421 (Acarbose) on postprandial blood glucose, serum insulin, and triglyceride levels: dose-time-response relationships in man. Res Exp. Med. (Berl.) 175, 87-94 (1979).

31. Hillebrand, I., Boehme, K., Frank, G., Fink, H. \& Berchtold, P. The effects of the alpha-glucosidase inhibitor BAY g 5421 (Acarbose) on meal-stimulated elevations of circulating glucose, insulin, and triglyceride levels in man. Res. Exp. Med. (Berl.) 175, 81-86 (1979).

32. Kageyama, S., Nakamich, N., Sekino, H. \& Nakano, S. Comparison of the effects of acarbose and voglibose in healthy subjects. Clin. Ther. 19, 720-729 (1997).

33. Koytchev, R. Influence of acarbose on blood glucose and breath hydrogen after carbohydrate load with sucrose or starch. Arzneimittelforschung 59, 557-563 (2009)

34. O'Dea, K. \& Turton, J. Optimum effectiveness of intestinal alpha-glucosidase inhibitors: importance of uniform distribution through a meal. Am. J. Clin. Nutr. 41, 511-516 (1985).

35. Uttenthal, L. O., Ukponmwan, O. O., Ghiglione, M. \& Bloom, S. R. Acute and short term effects of intestinal alpha-glucosidase inhibition on gut hormone responses in man. Dig. Dis. Sci. 32, 139-144 (1987).

36. Heinz, G., Komjati, M., Korn, A. \& Waldhäusl, W. Reduction of postprandial blood glucose by the alpha-glucosidase inhibitor Miglitol (BAY $m$ 1099) in type II diabetes. Eur. J. Clin. Pharmacol. 37, 33-36 (1989).

37. Lembcke, B. F. et al. Inhibition of glycemic and hormonal responses after repetitive sucrose and starch loads by different doses of the alpha-glucosidase inhibitor miglitol (BAY m 1099) in man. Pharmacology 43, 318-328 (1991).

38. Scott, A. R. \& Tattersall, R. B. Alpha glucosidase inhibition in the treatment of non-insulin-dependent diabetes mellitus. Diabet. Med. 5, 42-46 (1988).

39. Kennedy, F. P., Miles, J. M., Heiling, V. \& Gerich, J. E. The effect of two new alpha-glucosidase inhibitors on metabolic responses to a mixed meal in normal volunteers. Clin. Exp. Pharmacol. Physiol. 14, 633-640 (1987).

40. Van de Laar, F. A., Lucassen, P. L. B. J., Akkermans, R. P., Van de Lisdonk, E. H., De Grauw, W. J. C. Alpha-glucosidase inhibitors for people with impaired glucose tolerance or impaired fasting blood glucose. Cochrane Database Syst. Rev. CD005061 (2006) https://doi.org/10.1002/14651858. CD005061.pub2.

41. Aoki, K. et al. Miglitol administered before breakfast increased plasma active glucagon-like peptide-1 (GLP-1) levels after lunch in patients with type 2 diabetes treated with sitagliptin. Acta Diabetol. 49, 225-230 (2012). 